\title{
LFV, Dark Matter and LHC Data in Different GUTs
}

\author{
Mario E. Gómez ${ }^{1}$ and Smaragda Lola ${ }^{2}$ \\ ${ }^{1}$ Departamento de Ciencias Integradas y Centro de Estudios Avanzados en Física Matemáticas y Computación, Campus El Carmen, \\ Universidad de Huelva, 21071 Huelva, Spain \\ ${ }^{2}$ Department of Physics, University of Patras, 26500 Patras, Greece
}

\begin{abstract}
We perform an analysis of the predictions of several supersymmetric Grand Unified Theories (GUTs) for Dark Matter and the LHC and compare the results with a possible Lepton Flavour Violating (LFV) signal, when the models are extended with a see-saw mechanism to explain neutrino data. We study unified theories based on $S U(5)$, flipped $S U(5)$ and $S U(4)_{C} S U(2)_{L} S U(2)_{R}$, enhanced with a Type-1 see-saw mechanism. Each GUT predicts different Dark Matter (DM) scenarios, which can be used to classify the resulting SUSY predictions and confront them with experimental searches. Our results indicate that LFV is a powerful tool that complements LHC and DM searches, providing significant insight into the sparticle spectra and neutrino mass parameters in different models.
\end{abstract}

Keywords: supersymmetry phenomenology

DOI: $10.31526 /$ ACP.BSM-2021.24

\section{INTRODUCTION}

Despite its success, the Standard Model (SM) is not considered a definitive theory of the fundamental interactions. Observational evidence, pointing to the existence of dark matter in the universe [1,2], the observed neutrino flavour oscillations [3] but also theoretical issues, such as the hierarchy problem, have motivated searches for extensions of the SM, in which it arises as a low energy approximation. Among the first SM extensions are Grand Unified Theories (GUTs) [4, 5, 6, 7, 8], based on a symmetry that unifies the three interactions contained in it. In addition, Supersymmetric (SUSY) extensions of the SM [9] predict unification of the three fundamental SM interactions at a high energy scale called $M_{G U T}$. At this scale it is possible to postulate that the $S U(3)_{c} x S U(2)_{L} x U(1)_{Y}$ arises after the breaking of a higher symmetry that unifies the three interactions and acts from $M_{G U T}$ to a scale $M_{X}$ where gravity also unifies with the other interactions. Enhancing the minimal SUSY extension of the SM with a GUT theory [10] can provide a framework to analyse the recent LHC results and dark matter searches [11, 12, 13]. In addition, if the theory is complemented with a mechanism to explain neutrino flavour oscillations [14], the models will predict flavour violation also in the charged sector $[17,18,19,20,21,22]$. The motivation for our work is to confront the latest results on these predictions [23] with the DM ones [24, 25, 26, 27, 28] and the current results of the LHC [29, 30]. Following the procedure of refs. $[31,32,33,34]$ we present an update of these results to the current experimental bounds and we refer the reader to the quoted papers for comprehensive explanations and a complete set of references.

\section{GUT MASS RELATIONS AND DARK MATTER.}

We consider unified theories (GUTs) based on SU(5), flipped $S U(5)(F S U(5))$ and $S U(4)_{c} S U(2)_{L} S U(2)_{R}(4-2-2)$. We assume that SUSY breaking occurs at a scale $M_{X}>M_{G U T}$, through a mechanism that generates flavour blind soft-terms. Between the two scales, renormalisation can induce flavor dependence on the soft terms, however particles belonging to the same representation still have common soft masses at $M_{G U T}$.

In terms of a common soft mass $m_{0}$ for scalar SUSY particles we assume that at $M_{G U T}$ particles belonging to a representation $r$ have this mass modified by a factor $x_{r}$. This fact accounts for the renormalisation effects from $M_{X}$ to $M_{G U T}$ and other possible flavour symmetries. Therefore

$$
m_{r}=x_{r} m_{0}
$$

while the trilinear terms are defined as:

$$
A_{r}=Y_{r} A_{0}, \quad A_{0}=a_{0} m_{0} .
$$

Here, $Y_{r}$ is the Yukawa coupling associated to the $r$ representation. We use the standard parametrisation, with $a_{0}$ being a dimensionless factor, which we consider as representation independent.

Then the $M_{G U T}$ values for the soft terms in every GUT are:

- SU(5): In this case, the multiplet assignments are as follows:

$$
\left(Q, u^{c}, e^{c}\right)_{i} \in \mathbf{1 0}_{i},\left(L, d^{c}\right)_{i} \in \overline{\mathbf{5}}_{i}, v_{i}^{c} \in \mathbf{1}_{i} .
$$




\begin{tabular}{|c|ccc|}
\hline SUSY parameters & $4-2-2$ & SU(5) & FSU(5) \\
\hline $100 \mathrm{GeV} \leq m_{0} \leq 10 \mathrm{TeV}$ & $0 \leq x_{u} \leq 2$ & $0 \leq x_{u} \leq 2$ & $0 \leq x_{u} \leq 2$ \\
$50 \mathrm{GeV} \leq m_{1 / 2}\left(M_{2}\right.$ in $\left.4-2-2\right) \leq 10 \mathrm{TeV}$ & $0 \leq x_{d} \leq 2$ & $0 \leq x_{d} \leq 2$ & $0 \leq x_{d} \leq 2$ \\
$-10 \mathrm{TeV} \leq A_{0} \leq 10 \mathrm{TeV}$ & $0 \leq x_{L R} \leq 2$ & $0 \leq x_{5} \leq 2$ & $0 \leq x_{5} \leq 2$ \\
$2 \leq \tan \beta \leq 65$ & & & $0 \leq x_{R} \leq 2$ \\
$-3000 \mathrm{GeV} \leq M_{3}$ (in 4-2-2) $\leq 10 \mathrm{TeV}$ & & & \\
\hline & & & \\
\hline
\end{tabular}

TABLE 1: Parameter ranges sampled in our scan of the parameter spaces of the GUT models under consideration.

The masses of SUSY scalar particles are then defined as:

$$
m_{10}=m_{0}, \quad m_{5}=x_{5} \cdot m_{10}, \quad m_{H_{u}}=x_{u} \cdot m_{10}, \quad m_{H_{d}}=x_{d} \cdot m_{10},
$$

and the $A$ terms are specified via a common mass scale:

$$
A_{10,5}=a_{0} m_{0}
$$

- Flipped SU(5) (FSU(5)): The particle assignments are:

$$
\left(Q, d^{c}, v^{c}\right)_{i} \in \mathbf{1 0}_{i},\left(L, u^{c}\right)_{i} \in \overline{5}_{i}, e_{i}^{c} \in \mathbf{1}_{i},
$$

and the parametrisation changes to

$$
m_{10}=m_{0}, \quad m_{5}=x_{5} \cdot m_{10} \quad m_{R}=x_{R} \cdot m_{10} \quad m_{H_{u}}=x_{u} \cdot m_{10} \quad m_{H_{d}}=x_{d} \cdot m_{10},
$$

where $x_{R}$ refers to the $\mathrm{SU}(2)$-singlet fields. As previously, the $A$ terms are specified as universal: $A_{0}=a_{0} \cdot m_{0}$.

- 4-2-2 symmetry: In this case, the symmetry imposes a relation among the GUT values of the gaugino masses:

$$
M_{1}=\frac{3}{5} M_{2}+\frac{2}{5} M_{3} .
$$

The electroweak MSSM doublets lie in the 10-dimensional representation with D-term contributions that split their soft masses: $m_{H_{u, d}}^{2}=m_{10}^{2} \pm 2 M_{D}^{2}$. In our notation:

$$
x_{u}=\frac{m_{H_{u}}}{m_{16}}, \quad x_{d}=\frac{m_{H_{d}}}{m_{16}},
$$

with $x_{u}<x_{d}$. In the left-right asymmetric 4-2-2 model, a new parameter is introduced:

$$
x_{L R}=\frac{m_{L}}{m_{R}}
$$

where $m_{L}$ is the mass of the left-handed sfermions (that preserve the definition of $m_{16}=m_{0}$ ), and $m_{R}$ is the mass of the corresponding right-handed one.

The relic density constraint [2]:

$$
\Omega_{\chi} h^{2}=0.1186 \pm 0.0031,
$$

imposes strong conditions on the DM candidate. These conditions allow a classification of the different LSP in models that can satisfy this bound. For instance, in the MSSM, the most common mechanisms to satisfy the relic density condition are:

- Higgsino DM: $h_{f}>0.1,\left|m_{A}-2 m_{\chi}\right|>0.1 m_{\chi}$. Here, the parameter $h_{f}$ is the higgsino fraction of the lightest neutralino mass eigenstate defined as $h_{f} \equiv\left|N_{13}\right|^{2}+\left|N_{14}\right|^{2}$ where $N_{i j}$ are the elements of the unitary mixing matrix that correspond to the higgsino mass states.

- $\tilde{\tau}-\chi_{1}^{0}$ coannihilations: $h_{f}<0.1,\left(m_{\tilde{\tau}_{1}}-m_{\chi}\right) \leq 0.1 m_{\chi}$.

- $A / H$ resonances: $\left|m_{A}-2 m_{\chi}\right| \leq 0.1 m_{\chi}$.

The relation of parameters imposed by SU(5) allows further coannihilation mechanisms:

- $\tilde{\tau}-\tilde{v}_{\tau}-\chi_{1}^{0}$ coannihilations: $h_{f}<0.1,\left(m_{\tilde{\tau}_{1}}-m_{\chi}\right) \leq 0.1 m_{\chi}, \quad\left(m_{\tilde{v}_{\tau}}-m_{\chi}\right) \leq 0.1 m_{\chi}$. 

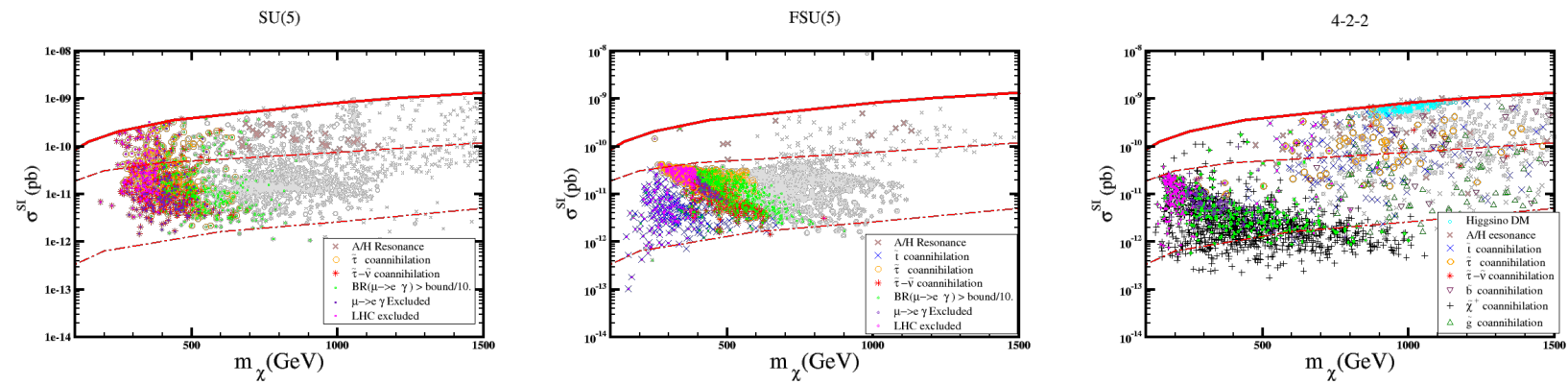

FIGURE 1: SI neutralino-nucleon cross section versus $m_{\chi}$ in SU(5), FSU(5) and the 4-2-2 escenarios. The solid lines correspond to the Xenon-1T bound [27], and the dashed and dot-dashed lines correspond to the projected sensitivities of the LZ [43] and DARWIN [42] experiments.

FSU(5) enables the stop coannihilations:

- $\tilde{t_{1}}-\chi_{1}^{0}$ coannihilations: $h_{f}<0.15,\left(m_{\tilde{t}_{1}}-m_{\chi}\right) \leq 0.1 m_{\chi}$.

Finally, the 4-2-2 model introduces a relation among gaugino masses and LR asymmetry that leads to new kinds of coannihilations:

- $\tilde{\chi}^{+}-\chi_{1}^{0}$ coannihilations: $h_{f}<0.1,\left(m_{\tilde{\chi}^{+}}-m_{\chi}\right) \leq 0.1 m_{\chi}$. In this case, the Higgsino component in the LSP is small, but the lightest chargino is light and nearly degenerate with the bino-like neutralino.

- $\tilde{g}-\chi_{1}^{0}$ coannihilations: $h_{f}<0.1,\left(m_{\tilde{g}}-m_{\chi}\right) \leq 0.1 m_{\chi}$. In this case, the gluino can be relatively light and nearly degenerate with the bino-like neutralino.

- $\tilde{b}-\chi_{1}^{0}$ coannihilations: $h_{f}<0.1,\left(m_{\tilde{b}}-m_{\chi}\right) \leq 0.1 m_{\chi}$. In this case, in the presence of LR asymmetry, the $\tilde{b}$ can be light and nearly degenerate with a bino-like neutralino.

Using the DM scenarios to classify the models, we can analyse the phenomenology of the different GUTs. Each class of model is represented by a specific mark, as indicated in Fig. 1. The values for the SUSY parameter ranges are given in Table 1. Since the signals depend on the particle properties of each model, we combine our analysis of LHC available data for every model under consideration. For that purpose, each model can be associated to a particular set of particle masses and decays, which are then compared with the generic analyses provided by the ATLAS and CMS collaborations [35, 36]. This task is simplified by using public packages like Smodels-v1.2.2 [37], which provides a powerful tool for performing a fast analysis of a large number of models $[38,39]$. Using this package, the theoretical models are compared with the existing LHC bounds. In each model the mass spectrum is generated using SoftSusy and the corresponding decay ratios are calculated using SUSY-HIT [40]. The cross-section information is then inserted in Smodels-v1.2.2 through a call to Pythia 8.2 [41]. We classify the models according to their LHC prospects and represent them in the plots with different color codes as follows:

- Magenta dots mark models excluded by the current LHC bounds;

- Colors different than grey correspond to models that can be compared with the LHC data and are not excluded;

- Grey corresponds to models that cannot be tested at the LHC, i.e., points that predict either processes with very low cross sections or topologies that are not tested at the LHC. Points excluded by $\mu \rightarrow e \gamma$ and LHC are also presented and will be discussed in the next section.

The DM predictions of the different GUT models can be seen in Fig. 1, where we display the spin-independent (SI) neutralinonucleon cross section as a function of the neutralino. The bound from the Xenon-1T experiment [27] is considered as an exclusion limit. We can observe that the projected DARWIN [42] experiment will cover all the SU(5) and FSU(5) models under consideration, while the 4-2-2 model still predicts models out of this range. The GUT mass relations of the different schemes predict different SUSY masses even for the same kind of coannihilations. The 4-2-2 model predicts larger SI cross sections even for neutralino masses $>1 \mathrm{TeV}$. We find in each model that the spin-dependent (SD) neutralino-neutron cross section is below the projected limit from the LZ [43] experiment.

\section{LEPTON-FLAVOUR MIXING EFFECTS AND SEE-SAW NEUTRINO MASSES}

The explanation of observed neutrino flavour oscillation requires an extension of our framework. In the see-saw scenarios, experimental data can be fitted by extending the MSSM with renormalizable interactions. We consider type-I see-saw, in which neutrino 

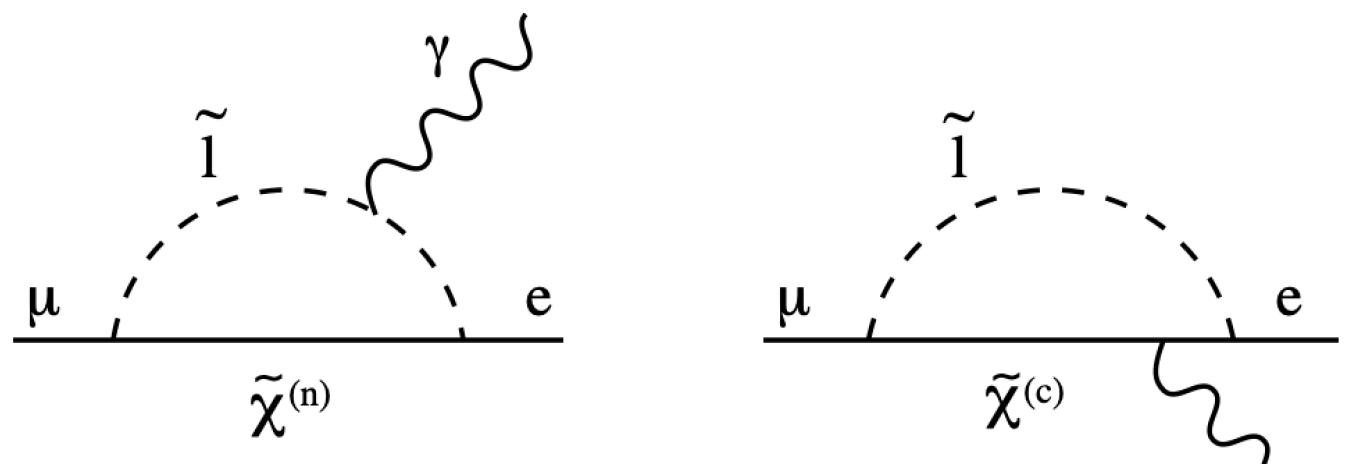

FIGURE 2: Generic Feynman diagrams for $\mu \rightarrow$ e $\gamma$ decay: I represents a charged slepton (left) or sneutrino (right), and $\tilde{\chi}^{(n)}$ and $\tilde{\chi}^{(c)}$ represent neutralinos and charginos respectively.

masses of the order of $0.1 \mathrm{eV}$ can be obtained introducing additional singlet RH neutrino at the $10^{13} \mathrm{GeV}$ scale. This mechanism can appear naturally in GUTs without affecting the running of the gauge couplings and, therefore, their unification.

The MSSM superpotential, supplemented with a type-I see-saw, becomes:

$$
W=W_{\mathrm{MSSM}}+Y_{v}^{i j} \epsilon_{\alpha \beta} H_{2}^{\alpha} N_{i}^{c} L_{j}^{\beta}+\frac{1}{2} M_{N}^{i j} N_{i}^{c} N_{j}^{c}
$$

where $W_{\text {MSSM }}$ is the MSSM superpotential and the $N_{i}^{c}$ are additional superfields that contain the three singlet (right-handed) neutrinos, $v_{R i}$, and their scalar partners, $\tilde{v}_{R i}$, and $M_{N}^{i j}$ denote the $3 \times 3$ Majorana mass matrix for the heavy right-handed neutrinos. The full set of soft SUSY-breaking terms is given by

$$
-L_{\text {soft,SI }}=-L_{\text {soft }}+\left(m_{\tilde{v}}^{2}\right)_{j}^{i} \tilde{v}_{R i}^{*} \tilde{v}_{R}^{j}+\left(\frac{1}{2} B_{v}^{i j} M_{N}^{i j} \tilde{v}_{R i}^{*} \tilde{v}_{R j}^{*}+A_{v}^{i j} h_{2} \tilde{v}_{R i}^{*} \tilde{l}_{L j}+\text { h.c. }\right),
$$

where $L_{\text {soft }}$ contains the MSSM soft SUSY-breaking masses, and $\left(m_{\tilde{v}}^{2}\right)_{j}^{i}, A_{v}^{i j}$ and $B_{v}^{i j}$ are the new soft SUSY-breaking parameters in the see-saw sector.

The see-saw mechanism yields three heavy neutral mass eigenstates that decouple at a high energy scale that we denote as $M_{N}$. Below this scale, the effective theory contains the MSSM plus a higher-dimensional operator that provides masses for the light neutrinos:

$$
W=W_{\mathrm{MSSM}}+\frac{1}{2}\left(Y_{v} L H_{2}\right)^{T} M_{N}^{-1}\left(Y_{v} L H_{2}\right)
$$

However, the running of the slepton masses from $M_{G U T}$ to $M_{N}$ is affected by the Dirac neutrino Yukawa coupling matrix $Y_{v}$ that can be of the same order as the fermion Yukawa couplings and therefore induce flavour changing terms on the slepton masses such that they cannot be diagonalised on the same basis as the lepton Yukawa $Y_{l}$. For instance, in such a basis and in the leading-log approximation [17] the slepton masses take the following form:

$$
\begin{aligned}
\left(m_{\tilde{L}}^{2}\right)_{i j} & \sim \frac{1}{16 \pi^{2}}\left(6 m_{0}^{2}+2 A_{0}^{2}\right)\left(Y_{v}^{+} Y_{v}\right)_{i j} \log \left(\frac{M_{\mathrm{GUT}}}{M_{R}}\right), \\
\left(m_{\tilde{e}}^{2}\right)_{i j} & \sim 0, \\
\left(A_{l}\right)_{i j} & \sim \frac{3}{8 \pi^{2}} A_{0} Y_{l i}\left(Y_{v}{ }^{\dagger} Y_{v}\right)_{i j} \log \left(\frac{M_{\mathrm{GUT}}}{M_{R}}\right) .
\end{aligned}
$$

leading to the prediction of LFV charged lepton decays at one loop through diagrams like the ones of Fig. 2. These processes, forbidden in the SM, are severely constrained by experiment. However, masses of the SUSY particles within the reach of the LHC can result to BR's of the order of the current experimental bounds [44]:

$$
\begin{aligned}
& B R(\mu \rightarrow e \gamma)<5.6 \times 10^{-13}, \\
& B R(\tau \rightarrow \mu \gamma)<4.4 \times 10^{-8} \\
& B R(\tau \rightarrow e \gamma)<3.3 \times 10^{-8} .
\end{aligned}
$$

In order to be able to make predictions for the LFV BR's mentioned above, in addition to the SUSY masses, we need to specify the see-saw parameters, namely the RH neutrino mass matrix and the product $Y_{v}^{\dagger} Y_{v}$.

Using the approach of $[20,45]$ a generic form for $Y_{v}$ that contains all neutrino experimental information can be obtained: 


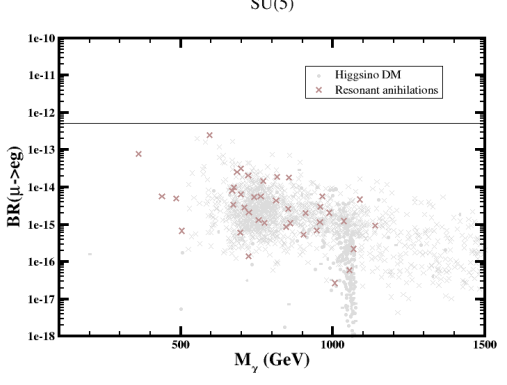

$\mathrm{SU}(5)$

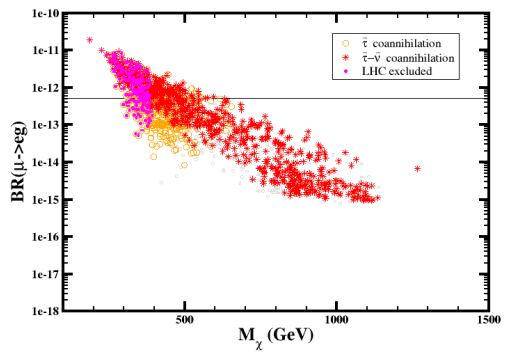

FSU(5)

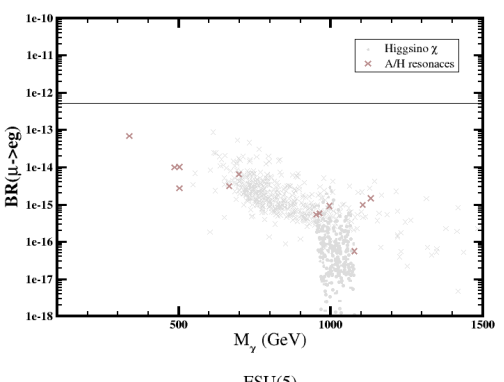

FSU(5)

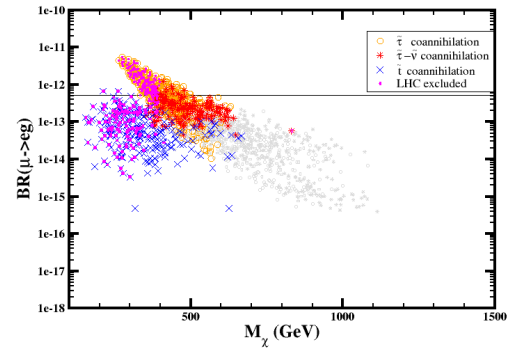

$4-2-2$

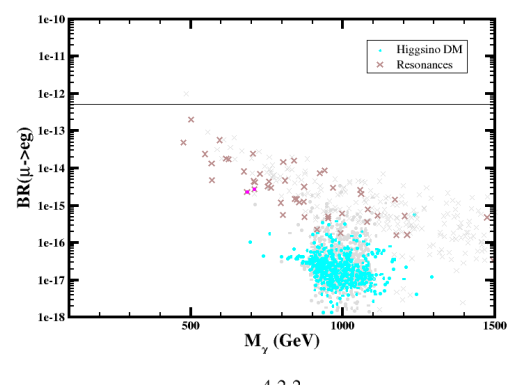

$4-2-2$

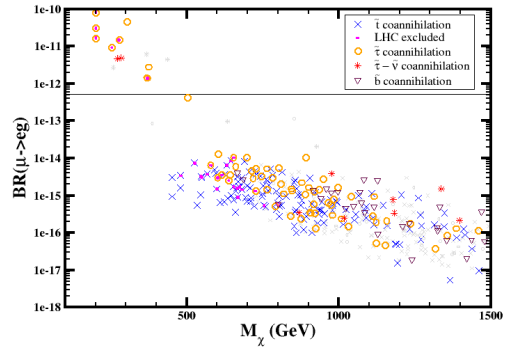

FIGURE 3: Prediction for $\mathrm{BR}(\mu \rightarrow e \gamma)$ vs $m_{\chi}$ for (from left to right) the SU(5), the FSU(5) and 4-2-2 models, in scenarios where sfermions coannihilate with the LSP. We use the same notation for the DM models as in Fig. 1. Models with parameters not detectable at the LHC are marked in grey, while excluded models are marked with purple dots. We assume $M_{N}=2.5 \cdot 10^{12} \mathrm{GeV}$ in all three cases.

$$
Y_{v}=\frac{\sqrt{2}}{v_{u}} \sqrt{M_{N}^{\delta}} O_{R} \sqrt{m_{v}^{\delta}} U^{\dagger},
$$

where $O_{R}$ is a general orthogonal matrix and $M_{N}^{\delta}$ and $m_{v}^{\delta}$ denote the diagonalized heavy and light Majorana neutrino mass matrices, respectively. In this basis, the matrix $U$ can be identified with the Pontecorvo-Maki-Nakagawa-Sakata (PMNS) matrix, $U_{\text {PMNS: }}$

$$
m_{v}^{\delta}=U^{T} m_{\mathrm{eff}} U .
$$

It is possible to obtain a successful prediction for the neutrino sector by imposing additional symmetries to the matrices $M_{R}$ and $Y_{v}$ [45]. However, textures that can solve the neutrino problem may give different predictions for LFV in the charged sector due to cancellations among partial contributions. We can get a simple but representative scenario by considering a common $\mathrm{RH}$ neutrino mass for the three species, $M_{N}$. In this case, using (19), we find

$$
Y_{v}^{\dagger} Y_{v}=\frac{2}{v_{u}^{2}} M_{R} U m_{v}^{\delta} U^{\dagger}
$$

Under the assumption of common masses for the heavy Majorana neutrinos, the LFV effects are independent of the matrix $O_{R}$ and hence the dependence on the possible textures for matrices $M_{R}$ and $Y_{v}$ is eliminated. The choice of $m_{v}^{\delta}=\operatorname{Diag}\left(1.1 \cdot 10^{-3}, 8\right.$. $\left.10^{-3}, 5 \cdot 10^{-2}\right) \mathrm{eV}$ is compatible with the observed neutrino oscillations. These masses can be obtained by combining values $M_{N}$ as large as $c \mathrm{GeV}$ with $Y_{v}$ couplings of order one. Here we assume $M_{N}=2.5 \times 10^{12} \mathrm{GeV}$ and with this choice we obtain values for the $\mathrm{BR}(\mu \rightarrow e \gamma)$ of the order of the current or projected experimental bounds for SUSY models that can be tested at the LHC.

We find that the values of $\operatorname{BR}(\tau \rightarrow \mu \gamma)$ in the framework under consideration are one order of magnitude below the $\operatorname{BR}(\mu \rightarrow e \gamma)$ ones, while the experimental bounds are five orders of magnitude apart. Keeping this in mind, we can present our LFV using as reference the $\mu \rightarrow$ er decay.

Following Fig. 3, we can discuss the LHC and charged LFV predictions of models by populating classes of points with different DM mechanisms that can be compared in the different unification scenarios:

- $\tilde{\tau}-\chi$ and $\tilde{\tau}-\tilde{v}-\chi$ coannihilation: These mechanisms both predict LFV and LHC signals within experimental reach. In the $\tilde{\tau}-\chi$ scenario, the lighter stau is determined by left-right mixing, and the $\tilde{\tau}-\tilde{v}-\chi$ is the limiting case where the $\tilde{\tau}_{1}$ is mainly left-handed. We should also take into account the fact that LFV is induced mainly in the left-left sector of the slepton mass matrix, due to the see-saw mechanism, therefore models with larger left-stau composition and smaller masses tend to have larger LFV decay rates. As seen in Fig. 3, this scenario is very interesting in SU(5) and FSU(5), since these models predict both LFV and LHC signals within experimental reach. In the case of 4-2-2 models, there is a left-right splitting of the sfermion soft masses, implying that points with stau coannihilations are more difficult to find. Furthermore, due to gaugino mass relations, the charginos and neutralinos can be heavier than two other GUTs, leading to lower $\operatorname{BR}(\mu \rightarrow e \gamma)$. 
$4-2-2$

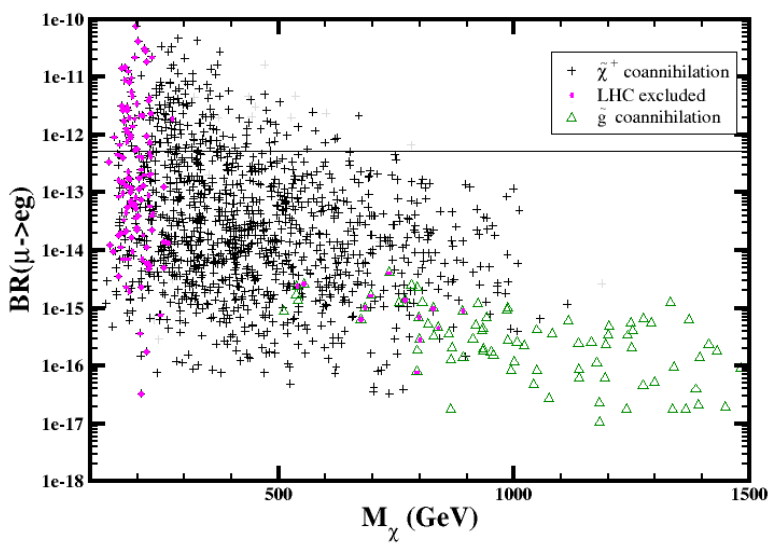

FIGURE 4: As in Fig. 3, for models with chargino and gluino coannihilations in the 4-2-2 GUT.

- $\tilde{t}-\chi$ coannihilation: Such models are present in the FSU(5) and in the 4-2-2 schemes, but with different predictions. In FSU(5), models with LSP masses up to $700 \mathrm{GeV}$ can predict ratios up to one order of magnitude below the current bound, whereas in 4-2-2 models the LSP mass can be larger, with $\mathrm{BR}(\mu \rightarrow e \gamma)$ two orders of magnitude below the experimental limit.

- A/H resonances and Higgsino DM: : These models are present in the three GUTs, and in all cases the predictions for LFV decays are below the current limits. However, in the Higgsino DM, the LSP composition is different in the three schemes; for instance, in the 4-2-2 model the LSP is almost a pure Higgsino and, even if $\mathrm{BR}(\mu \rightarrow e \gamma)$ is low, some model points can be tested at the LHC.

- $\tilde{\chi}^{+}-\chi$ and $\tilde{g}-\chi$ coannihilations: These DM classes appear only in the 4-2-2 case, due to the GUT relation on gaugino masses. As can be seen in Fig. 4, models with $\tilde{\chi}^{+}-\chi$ coannihilations have good detection prospects for both LFV decays and at the LHC. Points with $\tilde{g}-\chi$ coannihilation are still within the LHC reach, while the $\operatorname{BR}(\mu \rightarrow e \gamma)$ predictions are low.

\section{LHC SPECTROSCOPY AND LFV}

We combine the display of LFV predictions and LHC searches by selecting points on the planes of SUSY masses, as in Figs 5 and 6 . The solid red lines are obtained by combining the bounds imposed by LHC searches on simplified models. These bounds may not apply to our particular models, in which case the lines should not be understood as exclusion contours (though LHC excluded points would lie within at least one of these contours). Therefore, they can be used to confront the masses resulting from our models with the range of energies that are reached at the LHC for every SUSY particle. The LFV prediction is indicated by indigo crosses on points excluded by the limit on $\operatorname{BR}(\mu \rightarrow e \gamma)$, whereas the green crosses mark points that lie between the current bound and one order of magnitude below it.

Figs. 5 and 6 display LHC and LFV results on $m_{\tilde{g}}-m_{\chi}$ and $m_{\tilde{\chi}^{ \pm}}-m_{\chi}$ contour plots respectively. It is interesting to point out how the gaugino mass relations at GUT are reflected on the distribution of the models on the plots. In SU(5) and FSU(5), except for the Higgsino DM models, $m_{\tilde{g}}$ is almost proportional to $m_{\chi}$ and a similar relation holds for $m_{\tilde{\chi}^{ \pm}}$and $m_{\chi}$ in models where the lightest chargino is Wino-like. On the other hand, in the case of 4-2-2, these mass correlations follow some of the patterns described in Ref. [32]. In the case of SU(5) and FSU(5), all the excluded models are inside the red contour on the $m_{\tilde{g}}-m_{\chi}$ plots; we found that these models violate the constraint from the 0-lepton + jets $+Z_{T}$ channel $[46,47]$. This bound also affects most of the models excluded in the 4-2-2 case. However, in the lower planes of Fig. 6, we can find models inside the red line of the $m_{\tilde{\chi}^{ \pm}}-m_{\chi}$ plane. These correspond to models excluded by electroweak searches through the ATLAS multi-leptons $+\mathbb{T}_{T}$ channel [48]. Regarding LFV in SU(5) and FSU(5), we see that the models give rise to good detection prospects for chargino masses up to $1.5 \mathrm{TeV}$ while, in the case of 4-2-2, only models with chargino coannihilation present better prospects for LFV detection. In these cases, the masses reach the maximum value of $1 \mathrm{TeV}$ within our data range.

\section{CONCLUSIONS}

We combined the predictions of different SUSY unified theories for DM and the LHC in several GUT scenarios with the possibility of observing LFV in the charged sector. We considered scenarios with gaugino unification, such as SU(5) and FSU(5), and models where it can be relaxed, such as 4-2-2 models. 
SU(5)

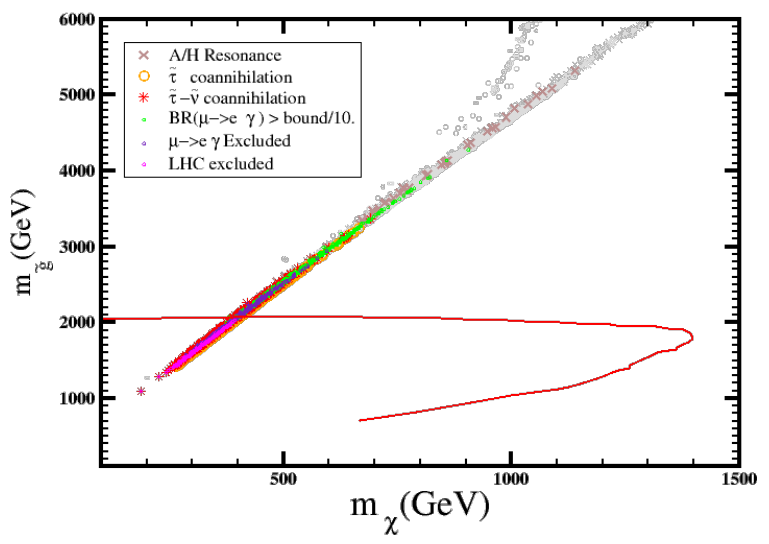

$\mathrm{SU}(5)$

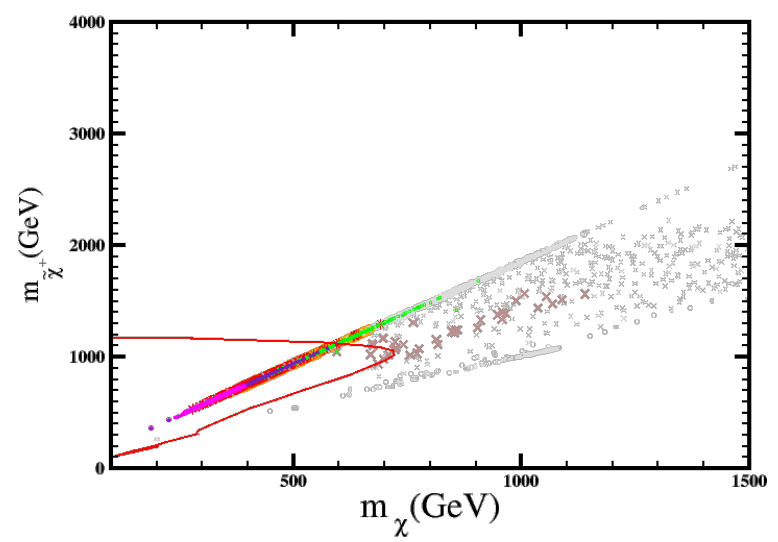

FSU(5)

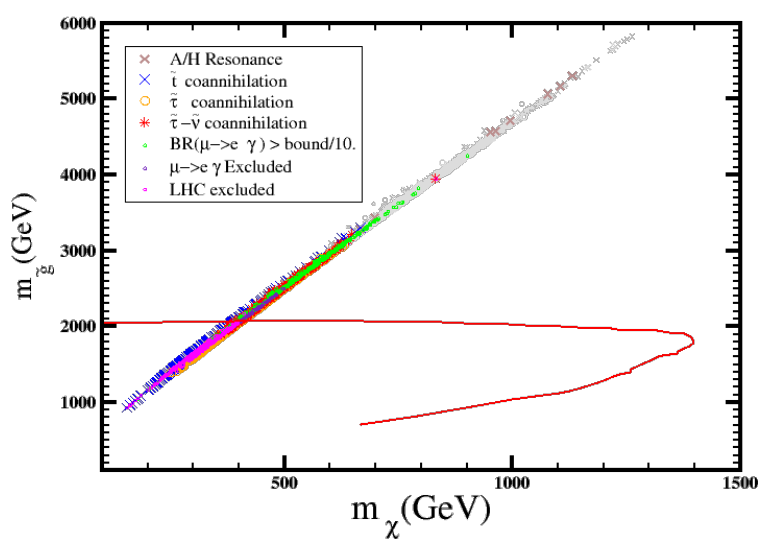

FSU(5)

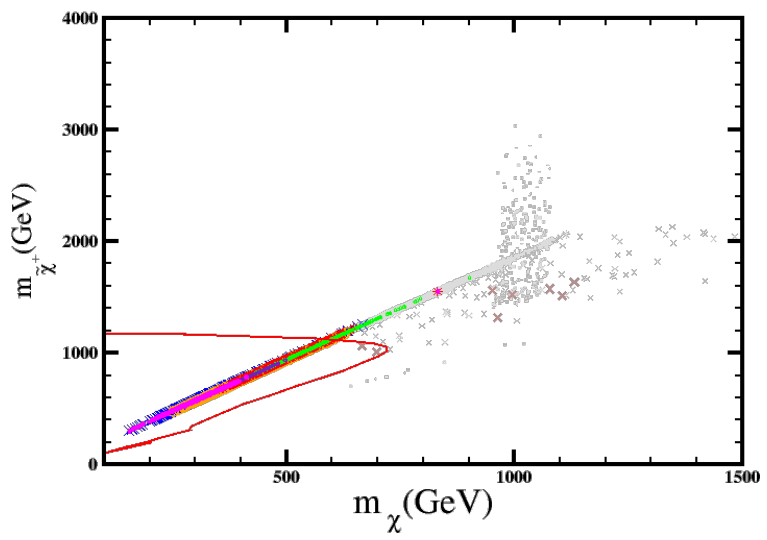

FIGURE 5: LHC prospects for the SU5 and FSU5 models. The points follow the notation of Figs. 3 and 4 . The meanings of the solid red lines are explained in the text. Indigo crosses indicate points excluded by the limit on $B R(\mu \rightarrow$ er), whereas the green crosses mark points that lie between the current bound and one order of magnitude below it. 
4-2-2

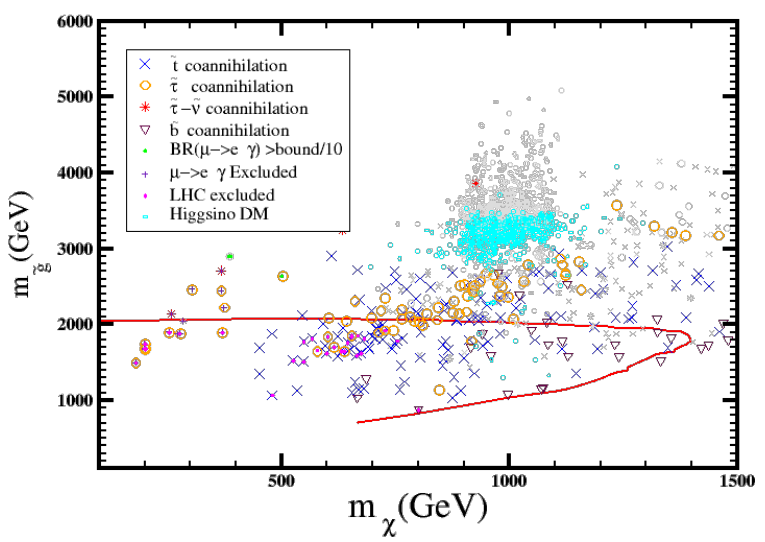

4-2-2

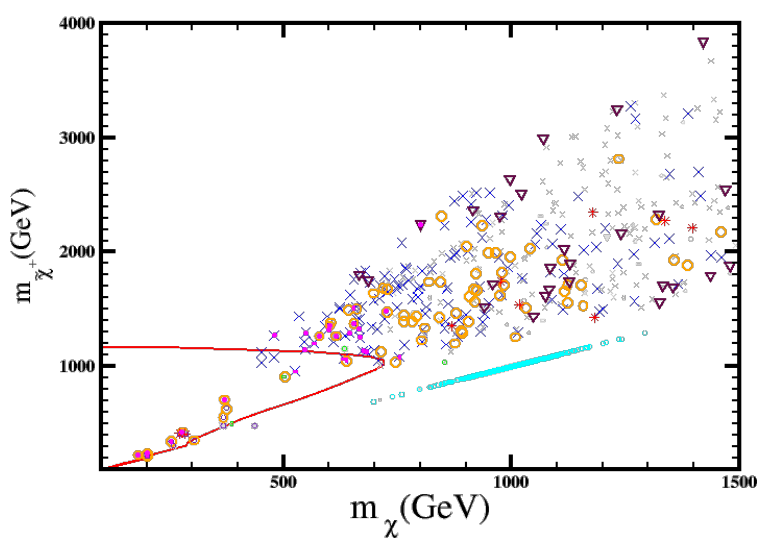

4-2-2

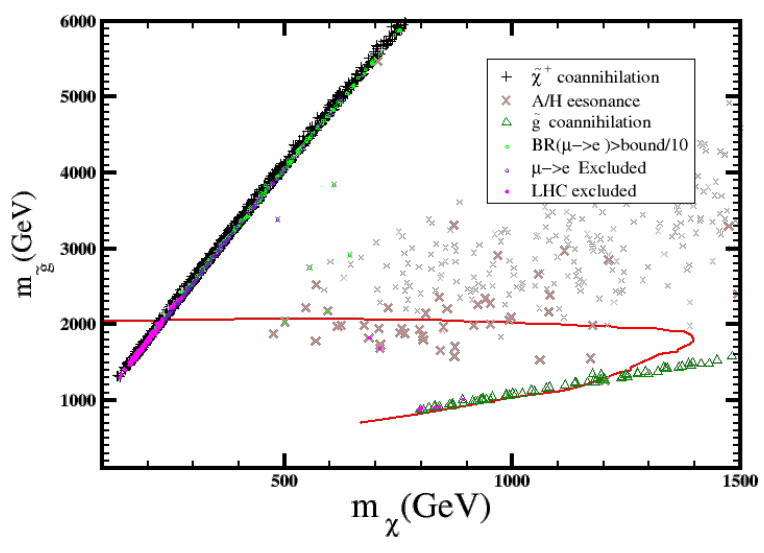

4-2-2

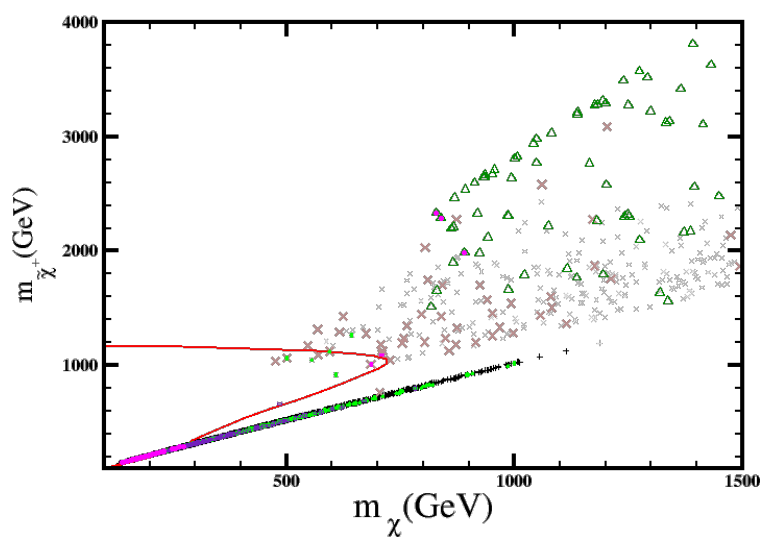

FIGURE 6: LHC prospects for the 4-2-2 model, following the notation of Fig. 5. For clarity of presentation, in the left panels we display predictions for models with sfermion coannihilations, whereas in the right panels we display the remaining cases. 
The three groups have distinctive LFV signatures, making it possible to link specific signatures in rare decays and colliders to the gauge and multiplet structure of the theory. In all three groups, coannihilations lead to higher rates for LFV, while resonant annihilations and higgsino dark matter are mostly not affected. Overall, in SU(5) and 4-2-2 it is easier to find annihilation models with good detection prospects both at the LHC and in LFV searches. Higgsino DM models do not predict detectable LFV. Still, it is interesting to note that the LSP composition is different in each scheme, yielding an almost pure Higgsino spectrum in the 4-2-2 model. Regarding LSP DM detection, the experiments are already reaching the sensitivity needed to cover most of the presented models, especially in SU(5) and 4-2-2 GUTs. In addition, the projected sensitivities of the LZ and DARWIN experiments will provide probes of models that are complementary to LFV searches, even for models that cannot be explored at the LHC.

Overall, our results indicate that LFV is a powerful tool that complements LHC and DM searches, and provides valuable information that can help identify optimal modes for future LHC searches. Moreover, not only does it distinguish clearly between various GUTs via the observability of different channels, but it can also provide significant insight into the respective sparticle spectra and neutrino mass parameters.

\section{ACKNOWLEDGMENTS}

The research of M.E.G. was supported by the Spanish MINECO, under grant PID2019-107844GB-C22.

\section{References}

[1] E. Komatsu et al. [WMAP Collaboration], Astrophys. J. Suppl. 192 (2011) 18 [arXiv:1001.4538 [astro-ph.CO]]. C. L. Bennett et al. [WMAP Collaboration], Astrophys. J. Suppl. 208 (2013) 20 [arXiv:1212.5225 [astro-ph.CO]].

[2] P. A. R. Ade et al. [Planck Collaboration], Astron. Astrophys. 571 (2014) A16 [arXiv:1303.5076 [astro-ph.CO]], Astron. Astrophys. 594 (2016) A13 [arXiv:1502.01589 [astro-ph.CO]].

[3] S. Fukuda et al. [Super-Kamiokande Collaboration], Phys. Rev. Lett. 86 (2001) 5656 [arXiv:hep-ex/0103033]; Phys. Rev. Lett. 86 (2001) 5651 [arXiv:hep-ex/0103032]; Phys. Lett. B 539 (2002) 179 [arXiv:hep-ex/0205075]; M. Apollonio et al. [CHOOZ Collaboration], Phys. Lett. B 466 (1999) 415 [arXiv:hep-ex/9907037]; Q. Ahmad et al. [SNO Collaboration], Phys. Rev. Lett. 87 (2001) 071301 [arXiv:nucl-ex/0106015]; Phys. Rev. Lett. 89 (2002) 011301 [arXiv:nucl-ex/0204008]; M. Ambrosio et al. [MACRO Collaboration], Phys. Lett. B 517 (2001) 59; G. Giacomelli and M. Giorgini [MACRO Collaboration], arXiv:hep-ex/0110021; K. Eguchi et al. [KamLAND Collaboration], arXiv:hep-ex/0212021.

[4] H. Georgi in "Particles and Fields" 1974, edited by C.E. Carlson, AIP Conference 34 Proceedings No. 23 , p. 575.

[5] H. Fritzsch and P.Minkowski, Annals Phys. 93 (1975) 193.

[6] J. C. Pati and A. Salam, Phys. Rev. D 10, 275 (1974).

[7] R.N. Mohapatra and J.C. Pati, Phys. Rev. D 11, 566 (1975); G. Senjanovic and R.N. Mohapatra, Phys. Rev. D 12, 1502 (1975); M. Magg, Q. Shafi and C. Wetterich, Phys. Lett. B 87, 227 (1979);

[8] G. Lazarides and Q. Shafi, Nucl. Phys. B 189, 393 (1981); T. W. B. Kibble, G. Lazarides and Q. Shafi, Phys. Lett. B 113, 237 (1982).

[9] H. E. Haber and G. L. Kane, Phys. Rept. 117 (1985), 75-263 doi:10.1016/0370-1573(85)90051-1

[10] J. R. Ellis, S. Kelley and Dimitri V. Nanopoulos, Phys. Lett. B249, 441, 1990 and Phys. Lett. B260, 131, 1991; Ugo Amaldi, Wim de Boer, and Hermann Furstenau. Phys. Lett., B260, 447, 1991; Paul Langacker and Ming-xing Luo, Phys. Rev. D44, 817, 1991; C. Giunti, C. W. Kim and U. W. Lee, Mod. Phys. Lett. A6, 1745, 1991.

[11] N. Okada, S. Raza and Q. Shafi, Phys. Rev. D 90 (2014) no.1, 015020 [arXiv:1307.0461 [hep-ph]].

[12] M. E. Gómez, Q. Shafi and C. S. Un, JHEP 07 (2020) no.07, 096 doi:10.1007/JHEP07(2020)096 [arXiv:2002.07517 [hep-ph]].

[13] P. Nath, Eur. Phys. J. ST 229 (2020) no.21, 3047-3059 doi:10.1140/epjst/e2020-000021-4

[14] P. Minkowski, Phys. Lett. B 67 (1977) 421; M. Gell-Mann, P. Ramond and R. Slansky, in Complex Spinors and Unified Theories eds. P. Van. Nieuwenhuizen and D. Freedman, Supergravity (North-Holland, Amsterdam, 1979), p.315 [Print-80-0576 (CERN)]; T. Yanagida, in Proceedings of the Workshop on the Unified Theory and the Baryon Number in the Universe, eds. O. Sawada and A. Sugamoto (KEK, Tsukuba, 1979), p.95; S. Glashow, in Quarks and Leptons, eds. M. Lévy et al. (Plenum Press, New York, 1980), p.687; R. Mohapatra and G. Senjanović, Phys. Rev. Lett. 44 (1980) 912; J. Schechter and J. W. F. Valle, Phys. Rev. D 22 (1980) 2227; Phys. Rev. D 25 (1982) 774.

[15] G. Lazarides, Q. Shafi and C. Wetterich, Nucl. Phys. B 181 (1981), 287-300 doi:10.1016/0550-3213(81)90354-0

[16] M. Cannoni, J. Ellis, M. Gómez and S. Lola, Phys. Rev. D 88 (2013) 7, 075005 [arXiv:1301.6002 [hep-ph]].

[17] J. Hisano, T. Moroi, K. Tobe and M. Yamaguchi, Phys. Rev. D 53 (1996), 2442-2459 doi:10.1103/PhysRevD.53.2442 [arXiv:hep-ph/9510309 [hep-ph]].

[18] M. Gómez, G. Leontaris, S. Lola and J. Vergados, Phys. Rev. D 59 (1999) 116009 [arXiv:hep-ph/9810291].

[19] J. Ellis, M. E. Gómez, G. Leontaris, S. Lola and D. Nanopoulos, Eur. Phys. J. C 14 (2000) 319 [arXiv:hep-ph/9911459].

[20] J. Casas and A. Ibarra, Nucl. Phys. B 618 (2001) 171 [arXiv:hep-ph/0103065].

[21] S. Antusch, E. Arganda, M. Herrero and A. Teixeira, JHEP 0611 (2006) 090 [arXiv:hep-ph/0607263].

[22] J. Ellis, M. Gómez and S. Lola, JHEP 0707 (2007) 052 [arXiv:hep-ph/0612292].

[23] A. M. Baldini et al. [MEG], Eur. Phys. J. C 76 (2016) no.8, 434 doi:10.1140/epjc/s10052-016-4271-x [arXiv:1605.05081 [hep-ex]].

[24] D. S. Akerib et al. [LUX Collaboration], Phys. Rev. Lett. 118 (2017) no.2, 021303 [arXiv:1608.07648 [astro-ph.CO]].

[25] E. Aprile et al. [XENON Collaboration], Phys. Rev. Lett. 119 (2017) no.18, 181301/ [arXiv:1705.06655 [astro-ph.CO]]; Phys. Rev. Lett. 121 (2018) no.11, 111302/ doi:10.1103/PhysRevLett.121.111302 [arXiv:1805.12562 [astro-ph.CO]].

[26] X. Cui et al. [PandaX-II Collaboration], Phys. Rev. Lett. 119 (2017) no.18, 181302 doi:10.1103/PhysRevLett.119.181302 [arXiv:1708.06917 [astroph.CO]].

[27] E. Aprile et al. [XENON Collaboration], Phys. Rev. Lett. 121 (2018) no.11, 111302 doi:10.1103/PhysRevLett.121.111302 [arXiv:1805.12562 [astroph.CO]].

[28] C. Amole et al. [PICO Collaboration], Phys. Rev. Lett. 118 (2017) no.25, 251301 [arXiv:1702.07666 [astro-ph.CO]]. 
[29] For a compendium of CMS searches for supersymmetry, see https://twiki.cern.ch/twiki/bin/view/ CMSPublic/PhysicsResultsSUS.

[30] For a compendium of ATLAS searches for supersymmetry, see https://twiki.cern.ch/twiki/bin/view/AtlasPublic/SupersymmetryPublicResults.

[31] M. Cannoni, J. Ellis, M. E. Gómez, S. Lola and R. Ruiz de Austri, JCAP 1603 (2016) 041 [arXiv:1511.06205 [hep-ph]].

[32] M. E. Gomez, S. Lola, R. Ruiz de Austri and Q. Shafi, Front. in Phys. 6 (2018), 127 doi:10.3389/fphy.2018.00127 [arXiv:1806.11152 [hep-ph]].

[33] M. E. Gómez, S. Lola, R. Ruiz De Austri and Q. Shafi, JHEP 10 (2018), 062 doi:10.1007/JHEP10(2018)062 [arXiv:1806.06220 [hep-ph]].

[34] J. Ellis, M. E. Gomez, S. Lola, R. Ruiz de Austri and Q. Shafi, JHEP 09 (2020), 197 doi:10.1007/JHEP09(2020)197 [arXiv:2002.11057 [hep-ph]].

[35] H. Okawa [ATLAS Collaboration], arXiv:1110.0282 [hep-ex].

[36] S. Chatrchyan et al. [CMS Collaboration], Phys. Rev. D 88 (2013) no.5, 052017 doi:10.1103/PhysRevD.88.052017 [arXiv:1301.2175 [hep-ex]].

[37] F. Ambrogi et al., doi:10.1016/j.cpc.2019.07.013 arXiv:1811.10624 [hep-ph].

[38] S. Kraml, S. Kulkarni, U. Laa, A. Lessa, W. Magerl, D. Proschofsky-Spindler and W. Waltenberger, Eur. Phys. J. C 74 (2014) 2868 doi:10.1140/epjc/s10052-014-2868-5 [arXiv:1312.4175 [hep-ph]].

[39] F. Ambrogi, S. Kraml, S. Kulkarni, U. Laa, A. Lessa and W. Waltenberger, Eur. Phys. J. C 78 (2018) no.3, 215 doi:10.1140/epjc/s10052-018-5660-0 [arXiv:1707.09036 [hep-ph]].

[40] A. Djouadi, M. M. Muhlleitner and M. Spira, Acta Phys. Polon. B 38 (2007) 635 [hep-ph/0609292].

[41] T. Sjöstrand et al., Comput. Phys. Commun. 191 (2015) 159 doi:10.1016/j.cpc.2015.01.024 [arXiv:1410.3012 [hep-ph]].

[42] J. Aalbers et al. [DARWIN Collaboration], JCAP 1611 (2016) 017 doi:10.1088/1475-7516/2016/11/017 [arXiv:1606.07001 [astro-ph.IM]].

[43] D. S. Akerib et al. [LUX-ZEPLIN Collaboration], arXiv:1802.06039 [astro-ph.IM].

[44] P.A. Zyla et al. [Particle Data Group], PTEP 2020 (2020) no.8, 083C01 doi:10.1093/ptep/ptaa104

[45] M. E. Gomez, S. Heinemeyer and M. Rehman, Eur. Phys. J. C 75 (2015) no.9, 434 doi:10.1140/epjc/s10052-015-3654-8 [arXiv:1501.02258 [hep$\mathrm{ph}]]$.

[46] A. M. Sirunyan et al. [CMS Collaboration], Phys. Rev. D 96 (2017) no.3, 032003 [arXiv:1704.07781 [hep-ex]].

[47] A. M. Sirunyan et al. [CMS Collaboration], Eur. Phys. J. C 77 (2017) no.10, 710 [arXiv:1705.04650 [hep-ex]].

[48] A. M. Sirunyan et al. [CMS Collaboration], JHEP 1803 (2018) 160 [arXiv:1801.03957 [hep-ex]]. 